\title{
A Conceptual and Methodological Synthesis on Modeling Ecological Niches and Geographical Distributions
}

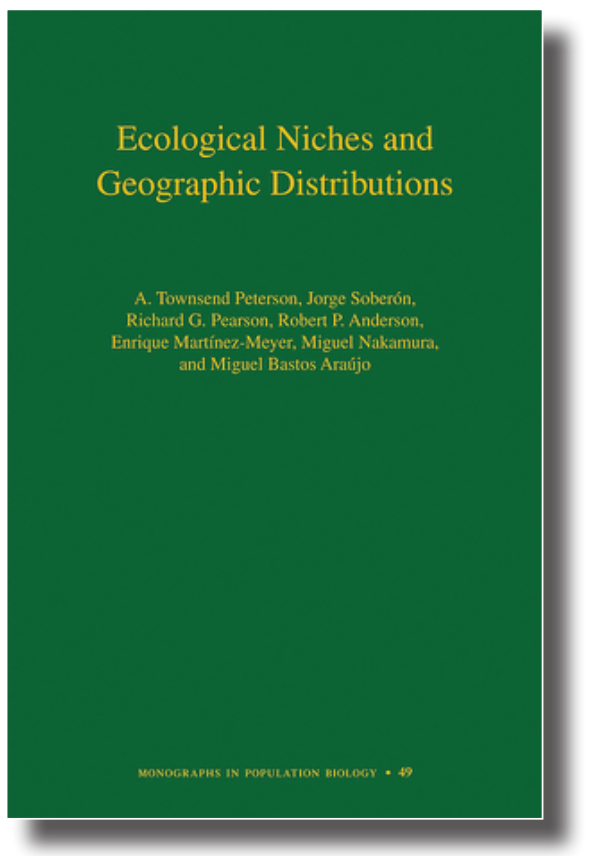

The increase availability of broad-scale records on species occurrences and environmental data, associated with the development of many computational and statistical tools to store, share and model these data, are the basis of the new field of "eco-informatics". These tools have also allowed modeling species ecological niches based on coupling their occurrence (and absence) with environmental data. These Ecological Niche Models (ENMs) - sometimes called Species Distribution Models (SDMs) or Bioclimatic Envelope Models (BEMs) - have allowed many different applications, both to understand how species' niches evolved and how they can be useful to understand species' geographical distributions, with important consequences for understanding their past and future range dynamics under global climate change and biotic movements (see Araújo \& Peterson (2012) and Peterson \& Soberón (2012) for a recent discussion on the exact meaning of ENMs, SDMs and the confusing terminology of this research field). Although citation analysis suggests that this research program is one of the most active ones in current ecology, biogeography and conservation biology, it is amazing how the program is loosely defined in many aspects and lacks conceptual and methodological integrations and consensus.
Peterson AT, Soberón J, Pearson RG, Anderson RP, MartínezMeyer E, Nakamura M \& Araújo MB. Ecological Niches and Geographic Distributions. Princeton University Press, Princeton, 2011, 314 p. ISBN: 978-0-691-13686-8.
The new book by A. Townsend Peterson, Jorge Soberón, Richard Pearson, Robert Anderson, Enrique Martínez-Meyer, Miguel Nakamura and Miguel Araújo comes exactly with the purpose of providing researchers and students with a more unified conceptual framework to model ecological niches and project geographic distributions, clarifying many issues that have been passionate discussed in the last years. Written by leading scientists in the field, who have been continuously developing and testing new methods, concepts and applications and thus provide important guidelines for dealing with ENMs and SDMs, the book is the number 49 of the prestigious series "Monographs in Population Biology" by Princeton University Press, which started with MacArthur \& Wilson's influential "Theory of Island Biogeography" published in 1967.

Peterson et al.'s (2011) book is divided into three sections (Theory, Practice and Applications), with a total of 16 chapters. Each section starts with a synthetic description of the chapters inside it, providing guidelines for understanding its conceptual logic. The first section on theory starts with two chapters containing a more conceptual discussion about ecological niches and species geographic distributions. Starting from Hutchinson's multivariate niche concept and discussing the many issues involved with this concept, such 
as the very definition of species' niche, variables related to niche and their scale, the existence of empty niches, the niche as requirements and impacts, Peterson et al. quickly move to an "operational" definition of niche by the multivariate E-space, delimited by "[...] scenopoetic variables that influence, at a geographic scale, the intrinsic and instantaneous growth rate of a species." (also referred to as the Grinnellian niche) (see Soberón 2007).

The link between E-space and geographical space (G-space) allows one to project niches and infer the geographical distribution of the species (and the other way around - see also Colwell \& Rangel 2009). However, going from E-space to G-space does not allow direct inference of geographical distributions; more is needed. Following the research tradition of Peterson's research group in the last years, the conceptual basis for this link is the so-called BAM diagram (from "Biotic", "Abiotic" and "Movement"), in which abiotically suitable areas, occupied ranges and invadable areas allows understanding species' presence and absences in the geographical space. Although it is still challenging to include processes beyond Grinnellian niches when modeling distributions in most practical studies, there is no doubt that BAM diagrams are a first heuristic tool when attempting to do this.

Then, a more practical section follows, and start by providing nice and useful guidelines to deal with data, both occurrence and environmental data, and models. The first chapter actually synthesizes the modeling steps, including data preparation, niche modeling per se, model projection and evaluation, and model transferability. Each of these steps is discussed in details in succeeding chapters.

The field of ENM and SDM has been somewhat plagued by many discussions about the algorithm used for to modeling ecological niches and inferring species distributions, which have been triggered by the heavily cited paper by Elith et al. (2006). These algorithms range from simple "bioclimatic envelope" models, such as BIOCLIM, up to complex machine learning methods, such as Artificial Neural Networks, Random Forests and Maximum Entropy (MaxEnt). Although it is trivial to think that more complex algorithms could provide better models, as measured by their high statistical goodness of fit, this is not necessarily true in all cases exactly because of data constraints and deviation from model assumptions, as well defined in BAM diagram. It is worthwhile to note that Peterson et al's book provide a refreshing perspective on this, focusing on overall modeling concepts and not on algorithms. There are actually about ten pages (out of 257 of main text) about the different algorithms that have been disputed in the literature. Although it would be nice to have a more detailed description of the methods (as provided in the other recent book by Janet Franklin, published in 2010), we understand the choice of the authors.

Beyond the discussion about model fit, a large portion of the "success" of an algorithm depends on the availability of fancy and friendly-used software. This is quite good, of course, and saves lots of time for everyone, but at the same time opens even more the possibility that people will start using complex algorithms without a minimum conceptual understanding of the methods and assumptions behind it Actually, this "Pandora effect" is typical of our times, and it is not uncommon to see students and researchers fitting a complex maximum entropy model (i.e., MaxEnt - see Elith et al. 2011) to model species' niches and project species' distributions, when they actually do not even understand the basic principles of a linear regression! In this sense, Peterson et al.'s book is rich in details about model calibration, evaluation and transferibility (and, by the way, it is important to note that many of the discussions about the performance of different algorithms depend on a deeper discussion on these more general modeling issues). We hope this book will fill the gap and solve the Pandora effect, stimulating researchers and students to think more clearly about the entire modeling process and not to focus on a given algorithm or method.

Finally, many different applications are discussed, including discovering biodiversity (i.e., the possibility of using ENMs to find new species and occurrences, as well as understand their geographic limits, in the context of Linnaean and Wallacean shortfalls), conservation planning and climate change, biological invasions and geographic of disease transmission, and the link between niches and evolutionary process. This latter link is heavily studied today, especially when dealing with the so-called "niche conservatism", an issue widely discussed today in a much broader macroecological and macroevolutionary context (see Wiens et al. 2010; Peterson 2011), but that arose in the ENM context (see Peterson et al. 1999).

The lack of conceptual and methodological rigor and the proliferation of different algorithms associated with alternative concepts and theoretical background, in some sense, are typical of a young research program in Ecology and Evolution. History of science seems to indicate that the development of a research program requires a gradual maturation so that consensus on methods and theories can be achieved, after many empirical analyses and data accumulation (as well as social factors, as death or retirement of old researchers and leaders). Only by leaving the mind open regarding new developments and empirical tests will allow a consensus to be achieved. But, as Carl Sagan (1934-1996) once said, one must "keep an open mind, but not so open your brains fall out...”. A balance must be achieved, and only a clear, honest and synthetic presentation of the theories allows this. We are sure that the book by Peterson et al. will be a landmark towards a more mature intellectual research program involving niche dynamics in evolutionary time and geographic space. Given the challenges that our society face to conserve biodiversity 
against so many threats, this is a more than welcome and timely contribution.

\section{References}

Araújo MB \& Peterson AT, 2012. Uses and misuses of bioclimatic envelope modelling. Ecology, 93:1527-1539. PMid:22919900. http://dx.doi.org/10.1890/11-1930.1

Colwell RK \& Rangel TF, 2009. Hutchinson's duality: The once and future niche. Proceedings of the National Academy of Sciences USA, 106:19644-19650. PMid:19805163 PMCid:2780946. http://dx.doi.org/10.1073/pnas.0901650106

Elith J et al., 2006. Novel methods improve prediction of species' distributions from occurrence data. Ecography, 29:129-151. http://dx.doi.org/10.1111/j.2006.0906-7590.04596.x

Elith J et al., 2011. A statistical explanation of MaxEnt for ecologists. Diversity and Distributions, 17:43-57. http:// dx.doi.org/10.1111/j.1472-4642.2010.00725.x

Franklin J, 2010. Mapping Species Distributions: Spatial Inference and Prediction. Cambridge: Cambridge University Press. http://dx.doi.org/10.1017/CBO9780511810602
Peterson AT, 2011. Ecological niche conservatism: A time-structured review of evidence. Journal of Biogeography, 38:817-827. http://dx.doi. org/10.1111/j.1365-2699.2010.02456.x

Peterson AT et al., 2011. Ecological Niches and Geographic Distributions. Princeton: Princeton University Press.

Peterson AT \& Soberón J, 2012. Species Distribution Modeling and Ecological Niche Modeling: Getting the Concepts Right. Natureza \& Conservação, 10(2): 102-107. http:// dx.doi.org/10.4322/natcon.2012.019

Peterson AT, Soberón J \& Sánchez-Cordero V, 1999. Conservatism of ecological niches in evolutionary time. Science, 285:1265-1267. PMid:10455053. http://dx.doi. org/10.1126/science.285.5431.1265

Soberón J, 2007. Grinnellian and Eltonian niches and geographic distributions of species. Ecology Letters, 10:1115-1123. PMid:17850335. http://dx.doi. org/10.1111/j.1461-0248.2007.01107.x

Wiens JJ et al., 2010. Niche conservatism as an emerging principle in ecology and conservation biology. Ecology Letters, 13:1310-1324. PMid:20649638. http://dx.doi. org/10.1111/j.1461-0248.2010.01515.x

José Alexandre Felizola Diniz-Filho \& Rafael Dias Loyola Departamento de Ecologia Universidade Federal de Goiás 
